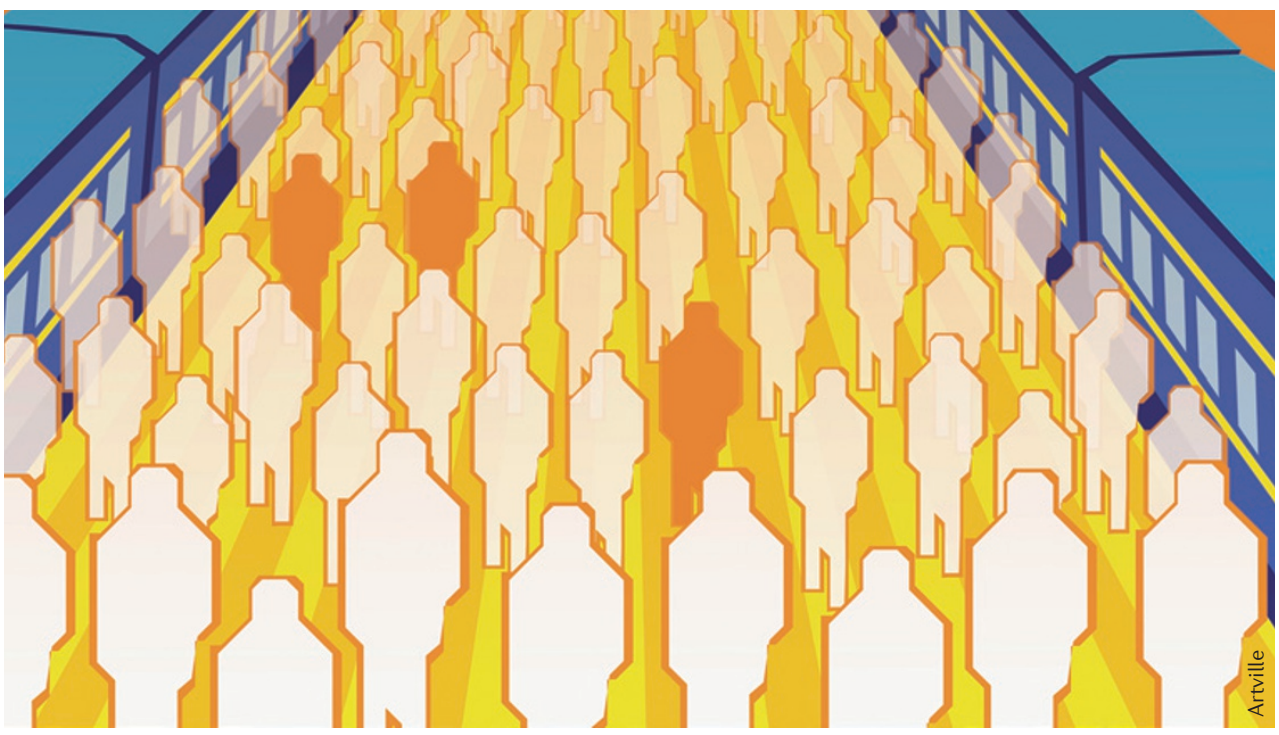

$\Rightarrow$ CANCER

\title{
Passenger deletions create cancer-specific Achilles heel
}

Cancer cells tend to shed tumour suppressor genes - and often delete neighbouring genes in the process. Now, reporting in Nature, DePinho and colleagues show that some of these 'passenger deletions' create cancer cell-specific vulnerabilities that can be targeted by new anticancer strategies.

The idea is based on the concept of collateral lethality - a situation in which members of gene families with housekeeping functions are specifically lost in cancer cells owing to their proximity to tumour suppressor genes, and pharmacological targeting of the remaining members of the gene family leaves cancer cells at a disadvantage compared to normal cells. To test the therapeutic exploitation of this concept, the authors examined passenger deletions in the cancer genome atlas data set for glioblastoma and homed in on enolase 1 (ENO1), which resides at the tumour suppressor locus 1 p36 and is deleted in $1-5 \%$ of glioblastomas. the conceptual enO1 encodes enolase, a ubiqu-
itously expressed enzyme that has framework of collateral lethality should be applicable to the development of highly personalized treatment regimens across many other cancer types an essential role in glycolysis. Model organisms such as Caenorhabditis elegans and Drosophila melanogaster have only one copy of the gene, and ENO1 deletion in these animals is lethal. By contrast, mammals have two more homologous genes ENO2, which is expressed in neural tissues, and ENO3, which is expressed in muscle tissues. ENO2 knockout mice are viable and fertile.

Out of 359 glioblastoma clinical samples, the authors found five that had homozygous deletions of ENO1. They also identified two glioblastoma cell lines with 1p36 deletions that included ENO1, as well as a control cell line in which most of the $1 \mathrm{p} 36$ locus was deleted, but not ENO1. Enolase-knockdown by short hairpin RNA directed against ENO2 induced profound growth inhibition of glioblastoma cell lines with the ENO1 deletion, but not in control cells. In vivo experiments with intracranially injected glioblastoma cells showed that a knockdown of ENO2 in ENO1-deleted cells completely abrogates their tumorigenic potential.

Further in vitro experiments used pharmacological inhibitors of enolase, which have so far mainly been investigated for antiparasitic purposes. Although enolase inhibitors such as phosphonoacetohydroxamate (PHAH), the most potent enolase inhibitor identified so far, have similar $\mathrm{IC}_{50} \mathrm{~s}$ for ENO1- and ENO2-encoded enolase, they are selectively toxic for ENO1-deleted cells. This was ascribed to the fact that enolase activity in these cells is $90 \%$ lower than in cells that express both ENO1 and ENO2. Further experiments showed a direct relationship between the level of enolase activity and sensitivity to PHAH. The authors found that treatment with this drug also resulted in a strong induction of $5^{\prime}$-AMP-activated protein kinase (AMPK), which was thought to be a protective stress response, leading them to speculate that a combination of PHAH and AMPK inhibitors might result in further toxicity for ENO1-deleted cancer cells.

These results provide proof-of concept for a new strategy that exploits genetically determined differences between cancer cells and normal cells. Given the large number of homozygous deletions across many cancer types (an estimated $11 \%$ of all proteincoding genes are deleted in human cancers), the authors hypothesize that the conceptual framework of collateral lethality should be applicable to the development of highly personalized treatment regimens across many other cancer types.

Alexandra Flemming

ORIGINAL RESEARCH PAPER Müller, F. et al.

Passenger deletions generate therapeutic

vulnerabilities in cancer. Nature 488, 337-342 (2012) 